TITLE:

\title{
Persistence of chaos in a time- delayed-feedback controlled Duffing system
}

\section{$\operatorname{AUTHOR}(S):$}

Yamasue, K; Hikihara, T

\section{CITATION:}

Yamasue, K ... [et al]. Persistence of chaos in a time-delayed-feedback controlled Duffing system. PHYSICAL REVIEW E 2006, 73(3): 036209.

ISSUE DATE:

2006-03

URL:

http://hdl.handle.net/2433/39914

RIGHT:

Copyright 2006 American Physical Society 
PHYSICAL REVIEW E 73, 036209 (2006)

\title{
Persistence of chaos in a time-delayed-feedback controlled Duffing system
}

\author{
Kohei Yamasue and Takashi Hikihara \\ Department of Electrical Engineering, Kyoto University, Katsura, Nishikyo, Kyoto 615-8510, Japan \\ (Received 8 April 2005; revised manuscript received 27 January 2006; published 14 March 2006)
}

\begin{abstract}
This paper concerns global phase structures of a time-delayed-feedback controlled two-well Duffing system. The remains of a global stretch and fold structure along an unstable manifold, which develops from an unstable fixed point in function space, reveals that the global chaotic dynamics is inherited from the original system by the controlled system. The remains of the original chaotic dynamics causes a highly complicated domain of attraction for target orbits and a long chaotic transient before convergence.
\end{abstract}

DOI: 10.1103/PhysRevE.73.036209

PACS number(s): $05.45 . \mathrm{Gg}$

\section{INTRODUCTION}

For more than a decade, extensive studies on controlling chaos have been carried out in the field of nonlinear dynamics [1]. With introduction of the sophisticated idea by Ott et al. [2], several advanced methods have been designed. These methods intend to change the chaotic motion of a system to a periodic one by stabilizing unstable periodic orbits embedded in chaotic attractors.

As one of the methods, time-delayed-feedback control [3] has attracted much interest of researchers up to the present. The strategy of this control method is to employ the difference signal between the present output signals and the past ones measured in a chaotic system. Control parameters are adjusted so that the difference signal converges to null when the controlled system is stabilized to a target unstable periodic orbit. Since the strategy is only relying on the time series of measurable output signals, the control method is a practical way to achieve continuous control of chaotic systems without the exact models of the systems nor complicated computer processing for reconstruction of the underlying dynamics. The feasibility of the control method has been experimentally verified already in a wide variety of fields including electronic circuits [4,5], laser systems [6], gas charge systems [7], mechanical oscillators [8], and chemical systems [9]. In addition, theoretical analysis has been performed based on stability analysis of the target unstable periodic orbits [10-16]. Among the results, the odd number condition is well known to give an inherent limitation concerning a class of unstable periodic orbits which cannot be stabilized by the strategy. The condition was first derived for discrete systems [17] and subsequently extended to continuous systems [11-13]. To overcome this limitation, Pyragas has recently improved the strategy [15].

On the other hand, there still remain open problems on the clarification of the control performance [14]. In particular, there has been no intrinsic discussion of the global dynamics of controlled systems and related control characteristics have not been clarified [18]. The global dynamics of a controlled system is governed by the global phase structure in function space, because the controlled system is described by differential difference equations [19]. The clarification of the global phase structure is important even in practice due to its direct relevance to the control characteristics, such as the domain of attraction for target orbits and transient behavior before convergence to them. As for the domain of attraction, the authors have recently reported that the boundary of the domain of attraction possibly has a self-similar structure [20]. It implies that the dynamics of controlled systems is governed by a complicated global phase structure that produces a sensitive dependence on initial conditions. Furthermore, the results are important from the practical point of view, because the sensitive dependence also implies that the success of control can be affected by the noise of the controlled system and the onset timing of control. The results have therefore shown that the global dynamics in function space should be considered for advanced use of the control method.

In this paper, we numerically discuss the global phase structure of a two-well Duffing system under time-delayedfeedback control. The two-well Duffing system is a model of first-mode vibration in the magnetoelastic beam system under sinusoidal forcing [21]. The control of chaos in the twowell Duffing system is an important subject of research relevant to the elimination of the chaotic vibration in mechanical systems. So far, the stabilization of chaos in a magnetoelastic beam system was experimentally achieved using time-delayed-feedback control [8]. The features of the phase propagation of the control signal were discussed in Ref. [18].

This paper consists of the following sections. Section II describes a mathematical model of the time-delayedfeedback controlled two-well Duffing system. Section III investigates the global phase structure of the controlled system by focusing on an unstable manifold. Section IV discusses the deterioration of the control performance based on the results in Sec. III.

\section{TWO-WELL DUFFING SYSTEM UNDER TIME- DELAYED-FEEDBACK CONTROL}

The Duffing system is a two-dimensional nonautonomous system originating in a model of synchronous machines [22]. Among a variety of its applications, the two-well Duffing system is derived as a model of the first-mode vibration in a magnetoelastic beam system under sinusoidal forcing [21]. The two-well Duffing system controlled by a scalar signal $u(t)$ is given as follows: 


$$
\frac{d}{d t}\left[\begin{array}{l}
x \\
y
\end{array}\right]=\left[\begin{array}{c}
y \\
-\delta y+\alpha x-\gamma x^{3}+A \cos \omega t
\end{array}\right]+\boldsymbol{b} u,
$$

where $x$ and $y$ denote the displacement and velocity of the system, respectively. $\boldsymbol{b}$ denotes a two-dimensional constant vector concerning coupling between the control signal and the state variables.

As Pyragas proposed in his paper, $u(t)$ is determined by the difference signal between the current output signal and the past one as follows [3]:

$$
u=K\left[g\left(x_{\tau}, y_{\tau}\right)-g(x, y)\right]
$$

where $\tau$ denotes the time delay and $K$ the feedback gain. $g(x, y)$ and $g\left(x_{\tau}, y_{\tau}\right)$ show a scalar output signal measured at the current time $t$ and the past time $t-\tau$, respectively. Since the control signal depends on only the time series of the output signal, the control method can be implemented in experimental systems without any exact models or detailed analyses of the underlying dynamics using the time series. The control performance has a crucial dependence on the time delay $\tau$ and the feedback gain $K$. The time delay is adjusted to the period of the target unstable periodic orbits embedded in a chaotic attractor. The control signal converges to null under precise adjustment, after the controlled system is stabilized on one of the target orbits. It is noted that some targeting method is additionally needed to avoid unexpected stabilization of an orbit which has the same period as the target orbits. The feedback gain governs the stability of target orbits under the precise adjustment of time delay. However, its systematic design has not been formulated at the present stage.

From the point of dynamical system theory, the two-well Duffing system controlled with delayed input should be treated as an infinite dimensional dynamical system due to the effect of time delay if $K \neq 0$. The temporal evolution of the controlled system is thus described by a discrete dynamical system whose phase space is a function space. The domain of attraction and transient behavior, which will be discussed later, are characterized by the global phase structure defined by this infinite dimensional system. On the other hand, the controlled system under $K=0$ is identical to the original two-well Duffing system. The controlled dynamics therefore coincides with the dynamics in the original twodimensional system.

The investigation is hereafter performed for the system parameters $\alpha=1.0$ and $\gamma=1.0$. The forcing frequency is fixed at $\omega=1.0$. The global dynamics under these parameters was summarized in Ref. [23]. The dynamics was classified with respect to the damping coefficient $\delta$ and the forcing frequency $A$. $\delta$ and $A$ are here fixed at 0.16 and 0.27 , respectively, so that the two-well Duffing system generates a chaotic attractor under the absence of the control signal. The chaotic attractor includes three unstable periodic orbits with period $2 \pi$ [23], as shown in Fig. 1 . Two of them, denoted by ${ }^{1} \mathrm{I}$ and ${ }^{1} \mathrm{I}$ ', are classified as inversely unstable and the remaining ${ }^{1} \mathrm{D}$ directly unstable by the location of their characteristic



FIG. 1. Unstable period-2 $\pi$ orbits embedded in original chaotic attractor [23]. ${ }^{1} I$ and ${ }^{1} I$ ' denote inversely unstable periodic orbits of the target, which are stabilized under sufficient large amplitude of feedback gain. ${ }^{1} D$ is a directly unstable periodic orbit, which cannot be stabilized because of the odd number condition. In Figs. 2(c), 2(d), 4, and 5 below, the notation ${ }^{1} I$ and ${ }^{1}{ }^{\prime}$ ' is replaced by ${ }^{1} S$ and ${ }^{1} \mathrm{~S}^{\prime}$, respectively, to reflect the stability change of the target orbits.

multipliers in the complex plane. ${ }^{1}$ The characteristic multipliers are obtained using the period $-2 \pi$ stroboscopic map. As target orbits for control, ${ }^{1} \mathrm{I}$ and ${ }^{1} \mathrm{I}$ ' are selected in this paper. ${ }^{1} \mathrm{I}$ and ${ }^{1} \mathrm{I}$ ' are easily stabilized by the control method. By measuring the velocity $y$, the control method is here implemented as follows:

$$
u=K\left(y_{2 \pi}-y\right) .
$$

This implementation is obtained by putting $\boldsymbol{b}=\left[\begin{array}{ll}0 & 1\end{array}\right]^{T}$, $g(x, y)=y$, and $\tau=2 \pi$ into Eqs. (1) and (2). It is noted that feedback of the velocity signal was employed for stabilization of the magnetoelastic beam [8].

Note that ${ }^{1} \mathrm{D}$ cannot be stabilized due to an inherent limitation of the control method. This limitation is characterized by a theoretical condition called the odd number condition as follows. A hyperbolic unstable periodic orbit cannot be stabilized by the original Pyragas method [3] and also by an extended one [5], if the orbit satisfies the odd number condition, namely, the orbit has an odd number of real characteristic multipliers greater than unity [13]. The odd number condition was first introduced by Ushio for discrete time control [17] and subsequently generalized for continuous time control by Nakajima et al. [11,13]. Just independently found essentially the same limitation [12]. The odd number condition obviously applies to any directly unstable orbit, because it has a unique real characteristic multiplier greater than unity. ${ }^{1} \mathrm{D}$ therefore cannot be stabilized under the con-

\footnotetext{
${ }^{1}$ This classification is based on Levinson's classification of periodic solutions in a system of class $D$, or a dissipative system for large displacement. A periodic orbit in the system is called inversely unstable, if its two characteristic multipliers $\rho_{1}$ and $\rho_{2}$ satisfy the relation $\rho_{2}<-1<\rho_{1}<0$. The periodic orbit is called directly unstable when its two multipliers have the relation $\rho_{1}>1>\rho_{2}>0$ [30].
} 

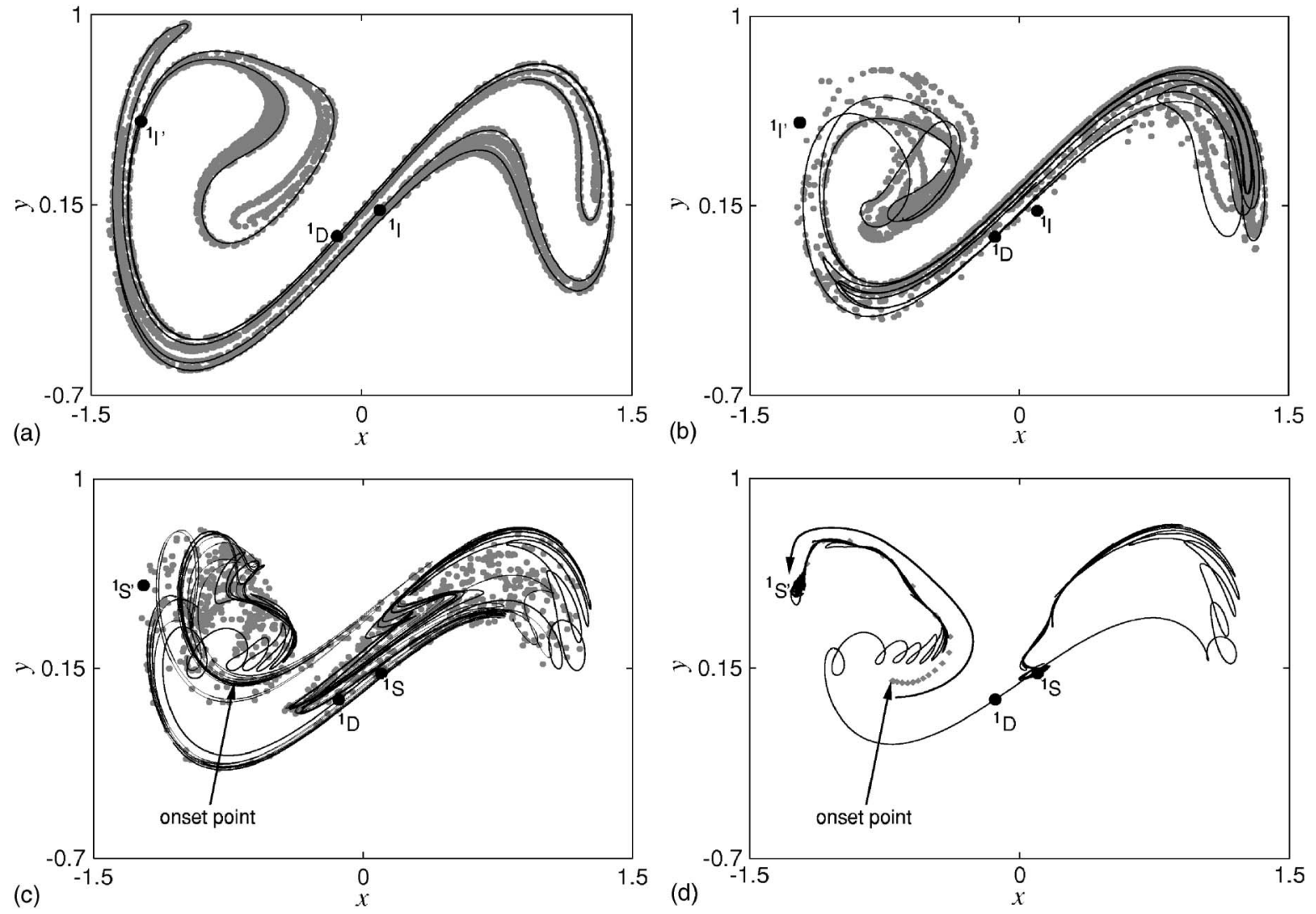

FIG. 2. Unstable manifold of ${ }^{1} \mathrm{D}$ (projection on a two-dimensional stroboscopic plane). In (a) and (b), the target orbits are unstable and the chaotic attractor is generated, as shown by gray stroboscopic points. In (c) and (d), the targets are stable and stroboscopic points show transient behavior before convergence to a target orbit. The notation of target orbits is changed to ${ }^{1} S$ and ${ }^{1} S^{\prime}$ because of their stability change. Arrows in (c) and (d) indicate the same stroboscopic point at which the control is activated.

trol method, and this fact motivates us to investigate the unstable manifold of ${ }^{1} \mathrm{D}$ in the next section.

\section{UNSTABLE MANIFOLD}

A key to understanding the controlled dynamics is to investigate dynamical structures which play a governing role in the global dynamics of the controlled system. We here focus on a one-dimensional global unstable manifold, which provides substantial information on the global phase structure in function space. ${ }^{2}$

As a starting point, the dynamics in the original twodimensional system is briefly described based on a literature summarizing the global dynamics of the two-well Duffing system [23]. The global dynamics of the two-well Duffing system is characterized by the presence of the directly unstable periodic orbit denoted by ${ }^{1} D$ in Fig. 1 . Since the stable and unstable manifolds of the orbit generate a homoclinic intersection in the cross section induced by stroboscopic mapping with period $2 \pi$, a chaotic invariant set exists in the original system. The closure of the unstable manifold coincides with the chaotic attractor, in which the target orbits and

\footnotetext{
${ }^{2}$ The same approach has been used in analysis of a laser system modeled by a differential difference equation. See Ref. [31].
}

${ }^{1} \mathrm{D}$ are embedded. Figure 2(a) shows the unstable manifold and the chaotic attractor for the original system, which are formally identical to the controlled system at zero feedback gain. One can easily confirm that the closure of the unstable manifold coincides with the chaotic attractor shown by gray stroboscopic points. The chaotic invariant set exists at this stage, because the unstable manifold of ${ }^{1} D$ transversely intersects the stable manifold of ${ }^{1} \mathrm{D}[21,23]$, as mentioned above. The regions of phase space are stretched and folded along the unstable manifold with its temporal evolution.

Once the control signal is activated under $K>0$, the original dynamics is perturbed by the control signal. The system under consideration changes from the original twodimensional system to the corresponding infinite dimensional one due to the presence of delayed input. On the other hand, the global phase structure itself is still governed by the unstable manifold of ${ }^{1} D$ because of the odd number condition. The odd number condition here implies that the ${ }^{1} D$ does not change its dynamical property under the control signal, that is, ${ }^{9} \mathrm{D}$ keeps a unique real characteristic multiplier greater than unity without any additional unstable multipliers for $K>0$. As a result, ${ }^{1} \mathrm{D}$ keeps the global unstable manifold tangent to the one-dimensional unstable subspace of ${ }^{1} D$ under control.

Figure 2(b) shows the unstable manifold for $K=0.3$. We note that the target orbits are still unstable in the controlled system and then the chaotic attractor is generated. One can 
clearly see that the unstable manifold inherits the global stretch and fold structure from the original unstable manifold for $K=0$. The two branches of the unstable manifold initially develop in opposite directions to each other. However, both branches are folded and then come close to ${ }^{1} \mathrm{D}$ again, parallel to themselves. The regions of the phase space are stretched around ${ }^{1} \mathrm{D}$ and folded around the left and right sides in Fig. 2(b) with temporal evolution, as was observed in Fig. 2(a) for $K=0$. The difference is that the unstable manifold is here projected from the function space to the original twodimensional stroboscopic plane. This is the reason that the unstable manifold appears to intersect itself.

As the feedback gain is further increased, the two target orbits become stable. However, the unstable manifold still keeps the stretch and fold structure. Figure 2(c) shows the unstable manifold for $K=0.75$ slightly over the threshold value for the stability change. The notation of the target orbits is here replaced by ${ }^{1} S$ and ${ }^{1} S^{\prime}$ to reflect this stability change. The two branches of the unstable manifold start from ${ }^{1} \mathrm{D}$ in the opposite direction from each other. However, they are folded and then come close to ${ }^{1} \mathrm{D}$ again parallel to the branches themselves. The branches further grow in the opposite direction from each other. The regions of phase space are stretched near ${ }^{1} \mathrm{D}$ and then folded around the left and right hand sides in Fig. 2(c) with temporal evolution. It implies that the phase space inherits the characteristics that produce the chaotic dynamics for $K=0$ and 0.3 , while the original chaotic attractor is destroyed because of the stability change of the target orbits.

With the same approach, we can confirm that further increase of feedback gain brings some global bifurcations which completely break the homoclinic intersection obtained in the original system. After these bifurcations, as shown in Fig. 2(d), the unstable manifold becomes quite simple compared with those in Figs. 2(a)-2(c). The unstable manifold in Fig. 2(d) therefore shows that the original chaotic invariant set disappears as the feedback gain is increased.

This section has discussed the global phase structure of the controlled system, focusing on the unstable manifold of ${ }^{1} \mathrm{D}$. The invariance of the qualitative features of the unstable manifold for increasing feedback gain has revealed that the the original global chaotic dynamics possibly remains in the controlled dynamics, even when the target orbits gain stability in the controlled system. In addition, we have observed that the original chaotic dynamics vanishes for high amplitude. These results indicate that the controlled dynamics exhibits crucially different behavior depending on the global phase structure. In the next section, influences on control performance are discussed.

\section{INFLUENCE ON CONTROL PERFORMANCE}

From the point of view of control, it is expected that controlled trajectories quickly converge to a target orbit selected preliminarily. However, when the chaotic dynamics remains in the controlled system, the trajectories wander irregularly between ${ }^{1} S$ and ${ }^{1} S$ ' along the unstable manifold even when the target orbits become stable. In Fig. 2(c), we can confirm this by the fact that a trajectory after onset of

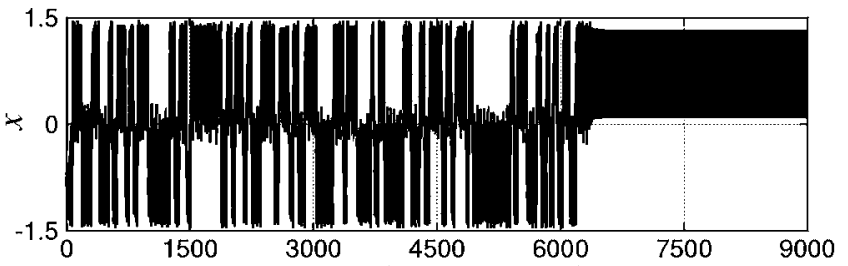

(a)

Elapsed time after onset time of control

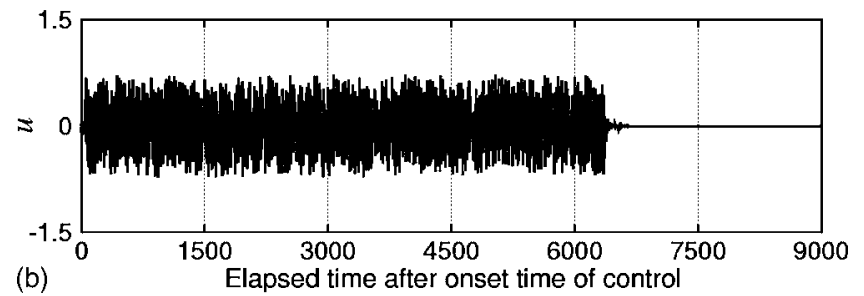

FIG. 3. Transient behavior in the time domain for $K=0.75$. Scale of time axis (not renormalized) is the same in (a) displacement and (b) control input. Controlled trajectory irregularly goes back and forth between two target orbits before final convergence to ${ }^{1} \mathrm{~S}$, corresponding to chaotic motion of stroboscopic points in Fig. 2(c). The same time scale is used in Fig. 6 below.

control is driven along the unstable manifold, as shown by stroboscopic points. Figure 3 shows the corresponding temporal change of displacement $x$ and control input $u$. One can see that the controlled trajectory irregularly goes back and forth between ${ }^{1} S$ and ${ }^{1} S^{\prime}$ many times, before it eventually converges to ${ }^{1} \mathrm{~S}$.

The steady states obtained by the control are not predictable because of this long and irregular transient behavior. Figure 4 shows a classification of stroboscopic points by the steady states. Each of the classified stroboscopic points here denotes the state of the system at the onset time of control, which was taken every $2 \pi$ period so that each of the chosen states is in the stroboscopic plane. Each of the stroboscopic points corresponds to a different initial condition including the state at the onset time. Different initial conditions for

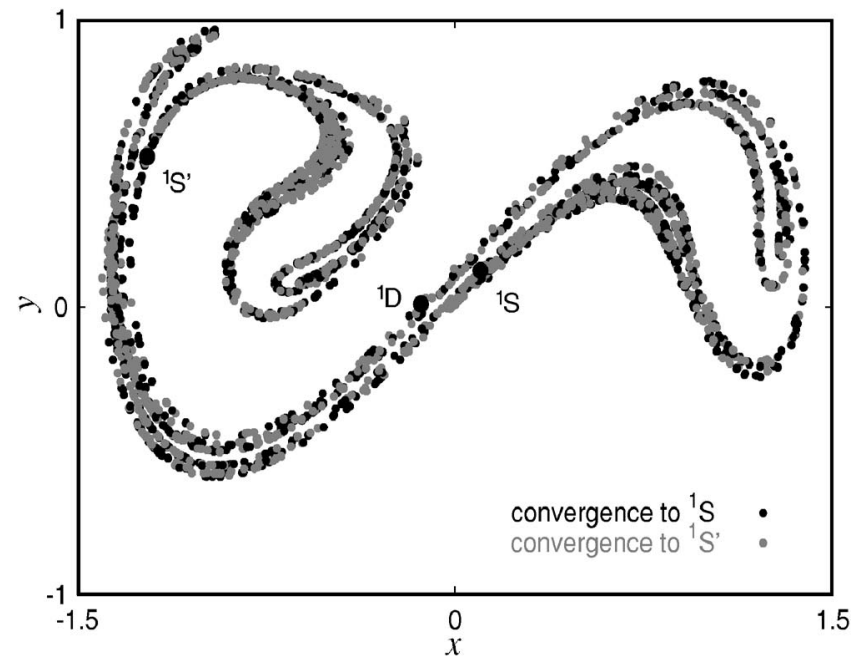

FIG. 4. Domain of attraction for target orbits at $K=0.75$. Black and gray points show convergence to ${ }^{1} S$ and ${ }^{1} S^{\prime}$, respectively. They are finely mixed with one another all over the original chaotic attractor. 




FIG. 5. Domain of attraction for target orbits at $K=1.1$. Black and gray points show convergence to ${ }^{1} S$ and ${ }^{1} S^{\prime}$, respectively. Most of the controlled trajectories converge to the target orbit in their initial side.

controlled dynamics were determined at every onset time, depending on a segment of a chaotic trajectory generated just before the onset. The controlled trajectories for the chosen initial conditions converged to either ${ }^{1} \mathrm{~S}$ or ${ }^{1} \mathrm{~S}^{\prime}$ after transient response. In Fig. 4, the stroboscopic points are classified with black and gray colors, which imply convergence to ${ }^{1} \mathrm{~S}$ and ${ }^{1} S^{\prime}$, respectively. The black and gray points are finely mixed with one another all over the original chaotic attractor. This implies that the steady states almost randomly alternate between ${ }^{1} S$ and ${ }^{1} S$ ' with onset timing of control and influence of external disturbance. In addition, since the two types of points are mixed even in the neighborhood of the target orbits, the controlled system is possibly stabilized to the target orbit at the opposite side from the other target, near which the control is activated. The targeting scheme based on linearization has therefore no possibility of effective convergence in the practical situation. These characteristics are obviously a disadvantage for engineering use of the control method. However, no detailed discussion of these characteristics has been obtained.

Corresponding to the simple global phase structure in Fig. 2(d), the domain of attraction becomes quite simple for $K=1.1$, as shown in Fig. 5. The classification implies that the targeting scheme is effective. Most of the trajectories converge to the target orbit located on their initial sides in the $x y$ plane, though there are some onset points leading the convergence to the opposite side. The trajectories go into two different sides in the process approaching ${ }^{1} \mathrm{D}$. The transient behavior is also much simpler than at $K=0.75$. Figure 6 shows that rapid convergence to a target orbit is achieved under high amplitude of the feedback gain.

We note that the classification adopted here has tested only initial conditions derived from a chaotic trajectory. In fact, the steady state for a chosen initial condition can be different, if the state of the uncontrolled system is partly modified in the time interval $\left[t_{0}-\tau, t_{0}\right]$ where $t_{0}$ is the onset time of control. Nevertheless, the classification has provided
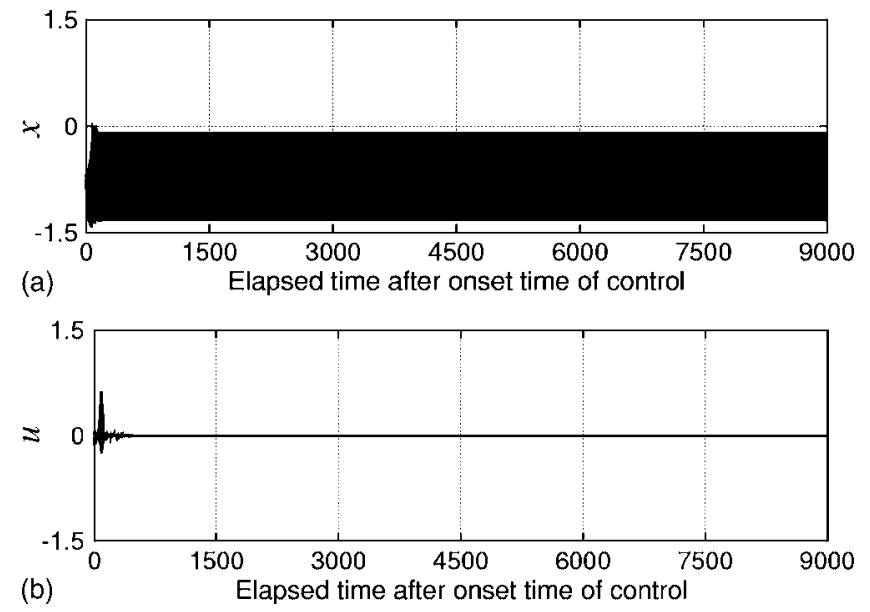

FIG. 6. Transient behavior for $K=1.1$. Time scale (not renormalized) is the same as in Fig. 3. Corresponding to the simple global structure in Fig. 2(d), the controlled trajectory quickly converges to ${ }^{1} \mathrm{~S}$ ' without any irregular behavior.

us substantial information on the structure of the domain of attraction. Since the uncontrolled system is chaotic, we can classify stroboscopic points densely plotted over the original chaotic attractor. This implies that the classification in this paper was performed for a system under no remarkable external disturbances which can modify the chosen initial conditions within the time interval.

In this section, we have shown that the transient behavior and domain of attraction accurately reflect the difference in the two global phase structures obtained in Sec. III. In particular, we have clarified that these control characteristics are significantly deteriorated when the original chaotic dynamics remains in the controlled system. It should be emphasized that one inevitably takes the global dynamics into account to estimate control performance. This is because the exact model of a chaotic system is often unknown and then control is possibly activated in the region where the linearization around a target orbit is not justified. The system after activation may exhibit long-term irregular behavior due to the remaining chaotic dynamics, even when the feedback gain is appropriately designed or optimized from the point of linear stability of target orbits. In other words, one should pay attention to the global dynamics besides linear stability in understanding the behavior of the controlled system and designing the control parameters.

Furthermore, we note that control of chaotic systems involves the destruction of chaotic attractors as its intrinsic nature. This is because the stability change of the target orbits embedded in a chaotic attractor can be achieved without any global bifurcations that disentangle the complicated phase structure originally producing the chaotic attractor. As a result, a long chaotic transient occurs as in the case of boundary crisis [24,25], while the different mechanism works behind the destruction. This implies, if chaos remains, that the success of controlling chaos is governed by some probabilistic law unless control is activated based on exact knowledge of the local dynamics around target orbits. It seems that the probabilistic law appears as the waiting time for the onset timing of control. 
We last recall that chaotic transients [24-26] and changes in the basin boundaries [27-29] have been connected to the collision and intersection of stable and unstable manifolds emanating from unstable periodic orbits especially in low dimensional systems. However, it is difficult, at least numerically, to identify the collision and intersection of manifolds in systems with time delay, because the stable manifolds have infinite dimension although the unstable manifolds keep finite dimensions in general. This is what made us consider only the unstable manifold of ${ }^{1} \mathrm{D}$; nevertheless, it has provided essential features of the global dynamics and related control characteristics.

\section{CONCLUSION}

In this paper, we have numerically discussed the global phase structure of the two-well Duffing system under time- delayed-feedback control. The focus on a one-dimensional unstable manifold gives substantial information about the global phase structure in function space. Our results indicate that the original chaotic dynamics possibly remains in the controlled system in particular near the threshold value of the stability change of the target orbits. The remains of chaos highly complicate the domain of attraction for the target orbits and causes long chaotic transient behavior before achieving convergence. These destructive influences on the control performance clearly show that the global dynamics in infinite dimensional space should be considered for further extension of the control method.

\section{ACKNOWLEDGMENTS}

This research is partially supported by the Ministry of Education, Culture, Sports, Science and Technology in Japan, The 21st Century COE Program No. 14213201.
[1] Handbook of Chaos Control, edited by H. G. Schuster (WileyVCH, Weinheim, 1999).

[2] E. Ott, C. Grebogi, and J. A. Yorke, Phys. Rev. Lett. 64, 1196 (1990).

[3] K. Pyragas, Phys. Lett. A 170, 421 (1992).

[4] K. Pyragas and A. Tamaševičius, Phys. Lett. A 180, 99 (1993).

[5] J. E. S. Socolar, D. W. Sukow, and D. J. Gauthier, Phys. Rev. E 50, 3245 (1994).

[6] S. Bielawski, D. Derozier, and P. Glorieux, Phys. Rev. E 49, R971 (1994).

[7] T. Pierre, G. Bonhomme, and A. Atipo, Phys. Rev. Lett. 76, 2290 (1996).

[8] T. Hikihara and T. Kawagoshi, Phys. Lett. A 211, 29 (1996).

[9] P. Parmananda, R. Madrigal, M. Rivera, L. Nyikos, I. Z. Kiss, and V. Gáspár, Phys. Rev. E 59, 5266 (1999).

[10] K. Pyragas, Phys. Lett. A 206, 323 (1995).

[11] H. Nakajima, Phys. Lett. A 232, 207 (1997).

[12] W. Just, T. Bernard, M. Ostheimer, E. Reibold, and H. Benner, Phys. Rev. Lett. 78, 203 (1997).

[13] H. Nakajima and Y. Ueda, Physica D 111, 143 (1998).

[14] W. Just, E. Reibold, K. Kacperski, P. Fronczak, J. A. Holyst, and H. Benner, Phys. Rev. E 61, 5045 (2000).

[15] K. Pyragas, Phys. Rev. Lett. 86, 2265 (2001).

[16] P. Hövel and J. E. S. Socolar, Phys. Rev. E 68, 036206 (2003).

[17] T. Ushio, IEEE Trans. Circuits Syst., I: Fundam. Theory Appl.
43, 815 (1996).

[18] T. Hikihara and Y. Ueda, Chaos 9, 887 (1999).

[19] J. K. Hale, Theory of Functional Differential Equations (Springer-Verlag, New York, 1977).

[20] K. Yamasue and T. Hikihara, Phys. Rev. E 69, 056209 (2004).

[21] F. C. Moon and P. J. Holmes, J. Sound Vib. 65, 275 (1979).

[22] G. Duffing, Erwungene Schwingungen bei Veränderlicher Eigenfrequenz und Ihre Technische Bedeutung (Druck und Verlag von Friedr. Vieweg \& Sohn, Braunschweig, 1918).

[23] Y. Ueda, H. Nakajima, T. Hikihara, and H. B. Stewart, in Dynamical Systems Approaches to Nonlinear Problems in Systems and Circuits, edited by F. M. A. Salam and M. L. Levi (SIAM, Philadelphia, 1988), pp. 128-137.

[24] C. Grebogi, E. Ott, and J. A. Yorke, Phys. Rev. Lett. 48, 1507 (1982).

[25] C. Grebogi, E. Ott, and J. A. Yorke, Physica D 7, 181 (1983).

[26] T. Tél, in Directions in Chaos, edited by B. L. Hao (World Scientific, Singapore, 1990), Vol. 3, pp. 149-211.

[27] F. C. Moon and G. X. Li, Phys. Rev. Lett. 55, 1439 (1985).

[28] C. Grebogi, E. Ott, and J. A. Yorke, Phys. Rev. Lett. 56, 1011 (1986).

[29] C. Grebogi, E. Ott, and J. A. Yorke, Physica D 24, 243 (1987).

[30] N. Levinson, Ann. Math. 45, 723 (1944).

[31] K. Green, B. Krauskopf, and K. Engelborghs, Physica D 173, 114 (2002). 